Supporting Information

\title{
Semi-transparent and Stable Solar Cell for Building Integrated Photovoltaic: The Confinement Effects of Polymer Gel Electrolyte inside Mesoporous Film
}

Huawei Zhou, ${ }^{*}, \dagger$ Jiawen Cui,, Junxue Guo, $\uparrow$ Siwen Tao, $†$ Xiaorui Gao, $†$ Meiqian Liu,,$\uparrow$ Miaomiao Wang, $\dagger$ Ning Yu, $\uparrow$ Xiaojun Wang, $\dagger$ Haoyu Gong, $\dagger$ Yanmin Li,, Ziang Wang, $\uparrow$ Tian Liu, $\uparrow$ Xun Sun, $\uparrow$ Yan Chen, $\uparrow$ Jie Yin, *,, and Xianxi Zhang, *,, Chunyang Zhang,t Yantao Shi*,t

$\dagger$ School of Chemistry and Chemical Engineering; College of Materials Science and Engineering; Shandong Provincial Key Laboratory/Collaborative Innovation Center of Chemical Energy Storage; Liaocheng University.

\# State Key laboratory of Fine Chemicals, School of Chemistry, Dalian University of Technology, Dalian 116024, China 


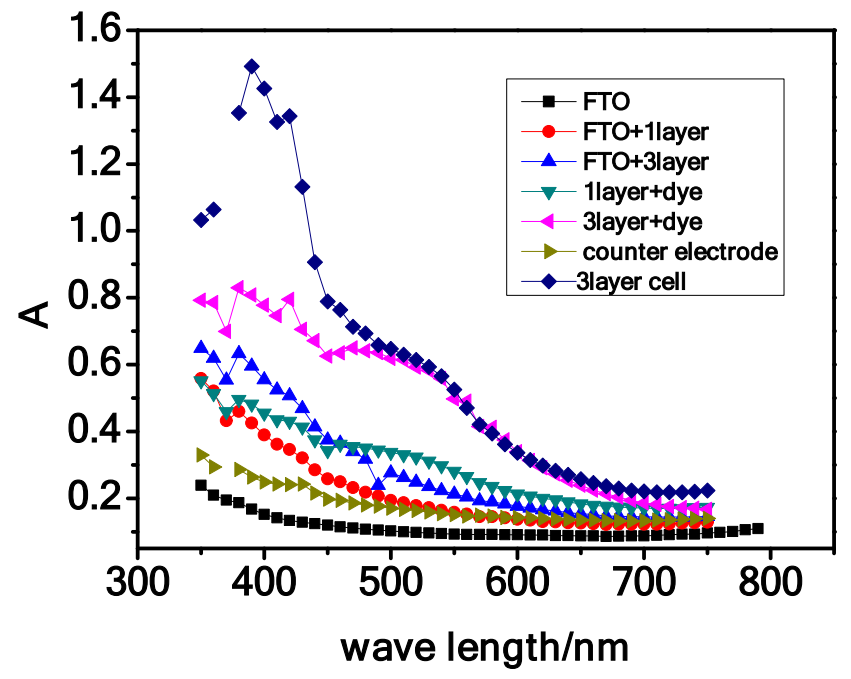

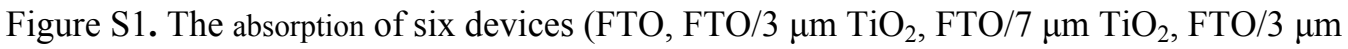
$\mathrm{TiO}_{2}+\mathrm{N} 719, \mathrm{FTO} / 7 \mu \mathrm{mTiO}_{2}+\mathrm{N} 719, \mathrm{Pt}$ counter electrode, and SQ-DSC)

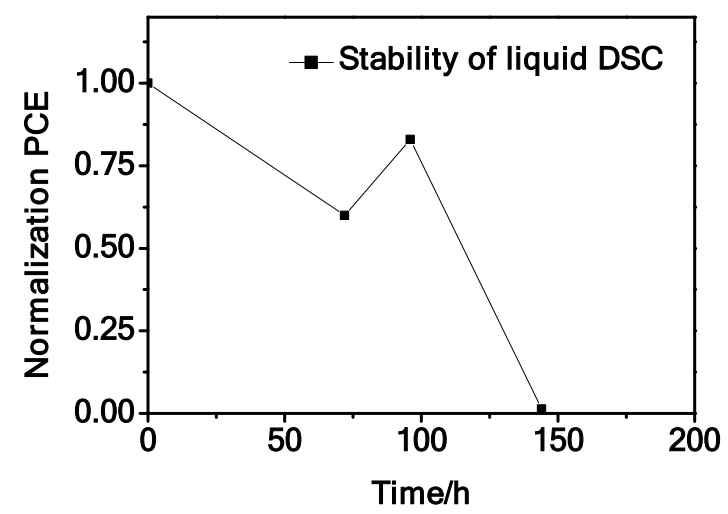

Figure S2 The stability of liquid DSC 